\title{
Terahertz Wave Generation via Nonlinear Parametric Process from $\varepsilon$-GaSe Single Crystals Grown by Liquid Phase Solution Method
}

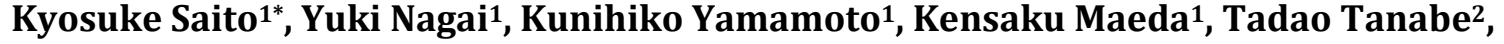 \\ Yutaka Oyama ${ }^{1}$ \\ ${ }^{1}$ Department of Materials Science, Graduate School of Engineering, Tohoku University, \\ Sendai, Japan \\ ${ }^{2}$ Institute of Multidisciplinary Research for Advanced Materials, Tohoku University, \\ Sendai, Japan \\ Email: ${ }^{*}$ k-saito@material.tohoku.ac.jp
}

Received 12 June 2014; revised 8 July 2014; accepted 1 August 2014

Copyright (C) 2014 by authors and Scientific Research Publishing Inc.

This work is licensed under the Creative Commons Attribution International License (CC BY). http://creativecommons.org/licenses/by/4.0/

(c) (i) Open Access

\section{Abstract}

Terahertz (THz)-wave generation has been conducted based on difference frequency mixing (DFM) process with phonon-polariton excitation of $\varepsilon$-GaSe single crystals implemented with liquid-phase solution growth using the temperature difference method under controlled vapour pressure for the first time. The type-eoo phase matching condition for the DFM process at around $10 \mathrm{THz}$ is satisfied by changing the incident angle into the crystal. The maximum conversion efficiency in the present DFG process is about $10^{-6} \mathrm{~J}^{-1}$ using a $0.1-\mathrm{mm}$-thick GaSe single crystal with the only $\varepsilon$ phase polytype, which can be greater than that of the commercially available Bridgman grown GaSe crystal including both $\varepsilon$ - and $\gamma$-phase polytypes.

\section{Keywords}

Terahertz Wave, Nonlinear Optics, Semiconductor Material, Liquid Phase Solution Growth

\section{Introduction}

Recently, terahertz (THz) waves, which locate above micro-wave frequency regions, have gathered much atten-

${ }^{*}$ Corresponding author.

How to cite this paper: Saito, K., et al. (2014) Terahertz Wave Generation via Nonlinear Parametric Process from $\varepsilon$-GaSe Single Crystals Grown by Liquid Phase Solution Method. Optics and Photonics Journal, 4, 213-218. 
tion because of their unique features, such as high transmittance through non-metallic materials, low photon energies that correspond to thermal energies of room temperature and weak molecular bonding, especially the hydrogen bonding. These features can be applied for non-destructive inspection, label-free identification of biomolecules, environmental polluting gas monitoring, and high speed wireless communication, etc. [1]-[3].

Monochromatic THz sources based on the nonlinear optical (NLO) effect such as optical parametric oscillation and difference-frequency generation (DFG) are promising methods due to their characteristic features such as high power, widely tuneable frequency and room temperature operation. NLO crystals based on the semiconductor material (GaAs [4], GaP [5] [6], and GaSe [7]-[9], etc.) are attractive materials because of their high NLO coefficient, high transparency in both infrared and $\mathrm{THz}$ regions, and the controlled growth methods to produce high quality single crystals. Especially, GaSe crystals are attractive materials because of the capability of high NLO coefficient $(d=54 \mathrm{pm} / \mathrm{V})[10]$ and $\mathrm{THz}$ wave generation in the wide frequency range which covers not only the $\mathrm{THz}$ frequency region but also mid-infrared regime $(0.1-100 \mathrm{THz})$ [9]. In addition, it is noted that the birefringence of bulk GaSe enables collinear phase matching conditions for THz generation via DFG process [7]-[9].

Most of commercially available GaSe crystals are grown using Bridgman method which introduces nonstoichiometric composition due to dissociation of Se atoms and introduction of point defect at the thermal equilibrium condition by high crystal growth temperature [11] [12]. Additionally, it has been shown that GaSe single crystals grown by this method possess several poly types. Therefore, not only the stoichiometry control but also the poly type control of GaSe single crystal is the serious problem for the fabrication of high quality NLO materials. We have successfully fabricated $\varepsilon$-GaSe single crystals for the first time using liquid phase solution growth based on temperature difference method under controlled vapour pressure (TDM-CVP) [13] [14]. This growth method can provide high quality NLO materials with low optical absorbance in both infrared and THz regions by precise stoichiometry control at lower growth temperature because the grown GaSe crystal possesses the single $\varepsilon$-phase polytype compared with that grown by Bridgman method which contains $\varepsilon$ - and $\gamma$-phase polytypes.

In this paper, it is shown that we conducted THz generation via DFG process using single polytype $\varepsilon$-GaSe single crystals grown by liquid phase solution method based on the TDM-CVP. Then, THz output characteristics were obtained by satisfying type-eoo phase matching conditions under the collinear phase matching conditions in the crystal.

\section{Experimental Section}

\subsection{Sample Preparation Procedure}

GaSe single crystal was prepared by the liquid phase solution method using TDM-CVP. Single poly type of $\varepsilon$-GaSe has been confirmed by X-ray diffraction (XRD) method and backward Raman scattering spectroscopy [13]. The input and output surfaces of the grown $\varepsilon$-GaSe were prepared by cleaving on (0001) plane by Scotch tape peeling-off method. Figure 1 shows typical optical microscope photograph (Nomarski differential interference contrast microscopy) of GaSe surface morphology after peeling. It is shown that the smooth surface morphology after the surface treatment can be obtained. The crystal thickness in $c$-axis direction was adjusted to be $0.1 \mathrm{~mm}$ by this peeling method, and anti-reflection coating was not applied to both input and output faces.

\subsection{Optical Setup for THz-Wave Generation}

Figure 2 illustrates the experimental setup for THz wave generation. Two ns-pulsed infrared pump light sources based on Cr:Forsterite laser (LOTIS TII Inc., 12 ns pulse duration, $500 \mathrm{MHz}$ linewidth, $10 \mathrm{~Hz}$ repetition rate) excited by a Q-switched 2ch. Nd:YAG laser (LOTIIS Inc.). Wavelength of the pump source was fixed at 1203 $\mathrm{nm}$, and signal source wavelength is able to tune in the range from $1249 \mathrm{~nm}$ to $1254 \mathrm{~nm}$ which corresponds to the difference frequency range from $9.4 \mathrm{THz}$ to $10.4 \mathrm{THz}$. The incident beams with pulse energy of each $5 \mathrm{~mJ}$ were collimated with diameter of $1 \mathrm{~mm}$ and combined in acollinear configuration. The collimated beams were incident onto (0001) plane of the $\varepsilon$-GaSe crystal. The polarizations of pump and signal sources were set to orthogonal configuration to satisfy a type-eoo phase matching (PM) condition in the collinearly phase matched DFG process. THz output radiation from the GaSe crystal was collected by off-axis parabolic mirrors and detected using a $4 \mathrm{~K}-\mathrm{Si}$ bolometer (Infrared Inc.). The infrared excitation beams were safely blocked by using a black polyethylene film located in front of the Si bolometer. 


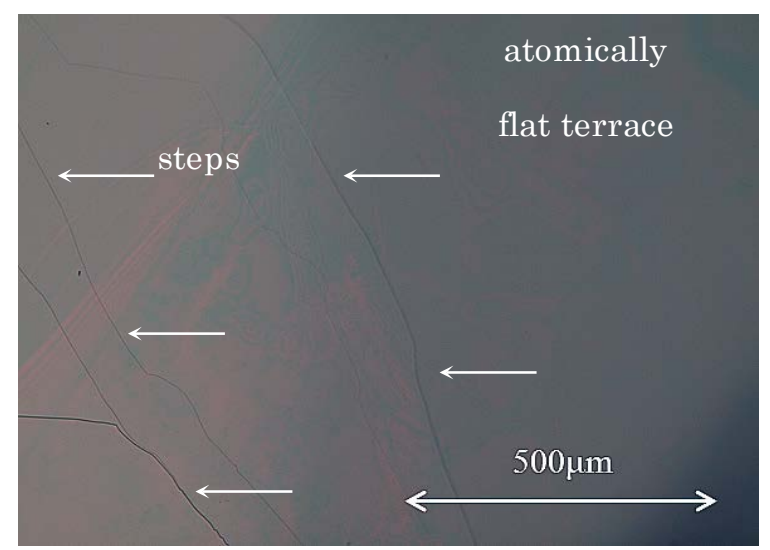

Figure 1. Optical microscope photograph of treated GaSe surface on (0001) plane after treated by Scotch-tape peeling method (Nomarski differential interference microscopic method).

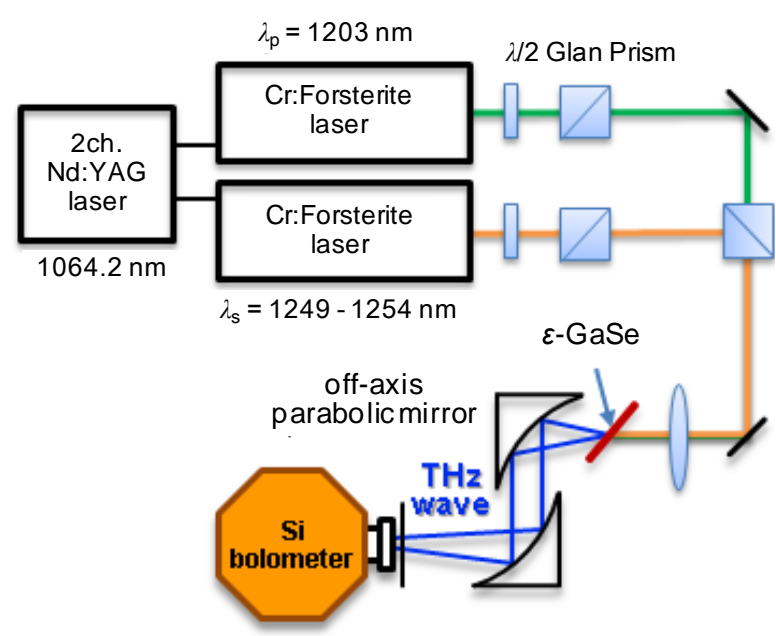

Figure 2. Schematic diagram of $\mathrm{THz}$ generation setup based on DFM process.

\section{Results and Discussion}

THz-wave generation was achieved on the basis of type-eoo PM condition using the $c$-axis cleaved $\varepsilon$-GaSe single crystal with 0.1-mm-long. Figure 3 shows external phase matching angle as a function of the difference frequency between two infrared sources. The external PM angles were measured as about 43,42 , and 41 degrees in the difference when the difference frequency was set to 9.41, 9.95, $10.41 \mathrm{THz}$, respectively. The type-eoo phase matching condition for THz-wave generation is expressed by,

$$
n_{p}^{(e)} \omega_{p}-n_{s}^{(o)} \omega_{s}=n_{T H z}^{(o)} \omega_{T H z},
$$

where $n_{p}^{(e)}$ and $n_{s}^{(o)}$ (i=s and THz) are extraordinary refractive index at the pump wavelength and ordinary refractive index at signal and produced THz-wave for GaSe crystal. The dispersion relationships of refractive indices for GaSe $n^{(e)}$ and $n^{(o)}$ obey following equations [15],

$$
\begin{gathered}
n^{(o)_{2}}=7.437+\frac{0.4050}{\lambda^{2}}+\frac{0.0186}{\lambda^{4}}+\frac{0.0061}{\lambda^{6}}+\frac{3.1436 \lambda^{2}}{\lambda^{2}-2193.8}+\frac{0.017 \lambda^{2}}{\lambda^{2}-262177.5577}, \\
n^{(e) 2}=5.760+\frac{0.3879}{\lambda^{2}}-\frac{0.2288}{\lambda^{4}}+\frac{0.1223}{\lambda^{6}}+\frac{0.4206 \lambda^{2}}{\lambda^{2} 17804},
\end{gathered}
$$




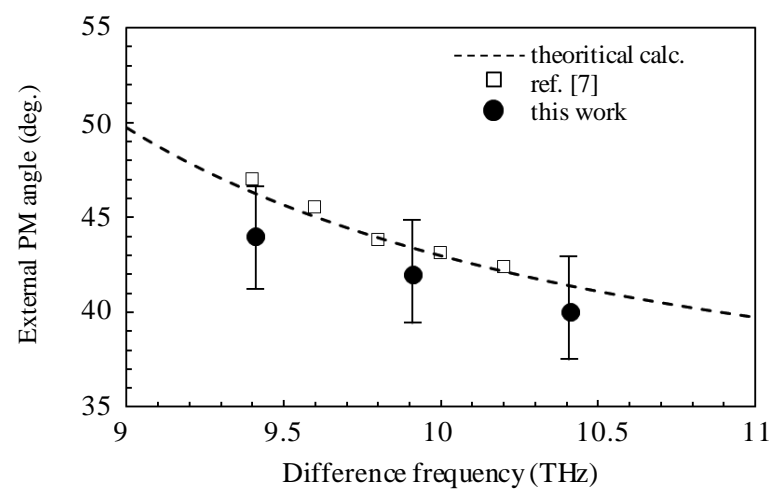

Figure 3. External phase matching (PM) angles as a function of the difference frequency of two infrared sources. Dashed line corresponds to the theoretical calculation for type-eoo PM, dots with error bar and open squares are experimental data obtained in this work and previous work using Bridgman method grown GaSe crystal (5-mm-long) in Ref. [7].

$$
\frac{1}{n^{(e) 2}(\theta)}=\frac{\sin ^{2} \theta}{n^{(e) 2}}+\frac{\cos ^{2} \theta}{n^{(e) 2}},
$$

where $\theta$ is angle between the propagation direction and $c$-axis of the GaSe crystal. Dashed curve in Figure 3 corresponds to the calculated results on the basis of Equations (1)-(4). The PM angles obtained from the experiment show slight deviation with and calculated results. The 100- $\mu \mathrm{m}$-thick GaSe crystal shows large acceptance angle for PM condition (shown as error bar in Figure 3) compared with 5-mm-thick GaSe crystal [7] (shown as open square in Figure 3).

Figure 4(a) shows the THz output power as a function of the external PM angle between $c$-axis of the crystal and the incident direction of the optical beams. For the type-eoo PM condition, monochromatic THz-waves with linewidth of about $0.5 \mathrm{GHz}$ were generated effectively above upper phonon-polariton branch at $9.41 \mathrm{THz}, 9.91$, and $10.41 \mathrm{THz}$, respectively. Maximum THz output power at each fixed frequency position decreased when the THz frequency increased because water vapour absorption exist around $10 \mathrm{THz}$ [15] [16]. Periodical THz output power variations in association with the PM angle change were observed in this figure. The reason of this phenomenon is expressed due to the interference of each incident infrared source and output THz-wave. The calculated result at difference frequency at $9.41 \mathrm{THz}$ by taking into account for the interference contribution was shown in Figure 4(b), which is consistent with the experimental result.

The THz output peak power was estimated with the calibrated Si bolometer and black polyethylene filter, which blocked the pump and signal beams. Power calibration of Si bolometer used was carried out by using black body radiation [17]. The highest THz peak power of $3 \mathrm{~mW}$ was achieved when the external phase matching angle was set to 44 degrees the difference frequency of THz-wave was set to $9.41 \mathrm{THz}$. The corresponding conversion efficiency was estimated to be about $1.2 \times 10^{-6} \mathrm{~J}^{-1}$ by using the 0.1 -mm-long crystal. This estimated conversion efficiency can be comparable with the $\mathrm{THz}$ output from the commercially available 2-mm-long GaSe crystal grown by Bridgman method (in the order of $10^{-7} \mathrm{~J}^{-1}$ ) [7]. The improvement of THz conversion efficiency can be supported by the decrease of absorption coefficient for the incident infrared sources associated with the energy state of deep level in the $\varepsilon$-GaSe single crystal [13] [14].

\section{Conclusion}

In conclusion, THz-wave generation via DFG process has been realized using pure $\varepsilon$-GaSe single crystals fabricated by liquid phase solution growth, using the temperature difference method under controlled vapour pressure (TDM-CVP). The type-eoo phase matching condition is satisfied by changing the incident angle into the crystal. The maximum conversion efficiency in the DFG process is about $10^{-6} \mathrm{~J}^{-1}$ using a 0.1 -mm-long $\varepsilon$-GaSe single crystal. Our growth method is able to provide high quality NLO crystals and develop the efficient monochromatic THz sources. 


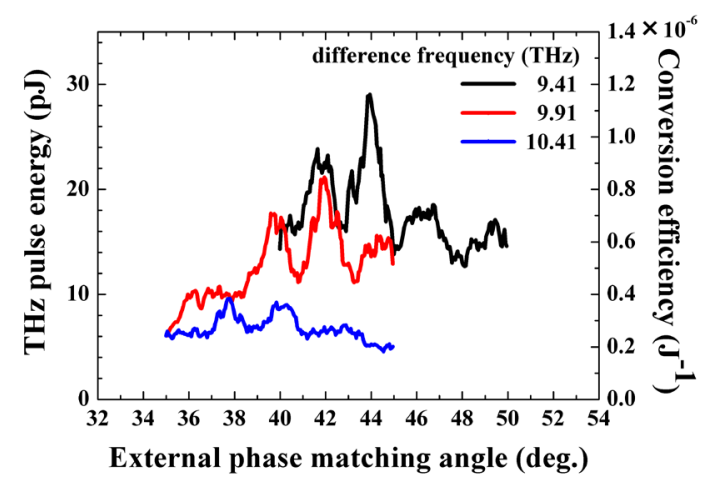

(a)

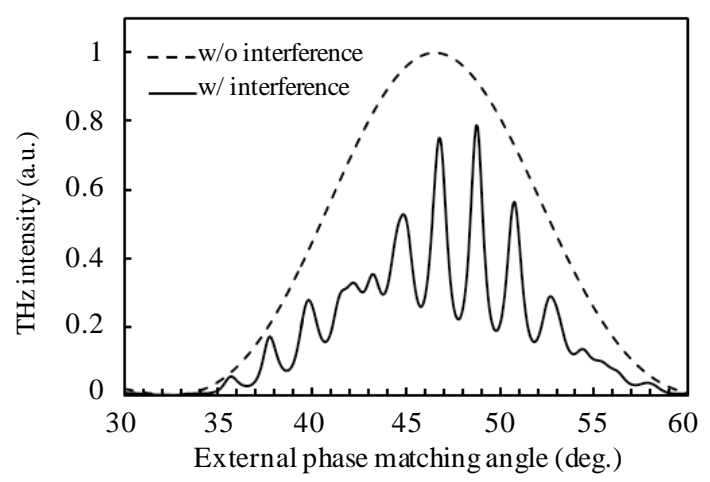

(b)

Figure 4. (a) THz output power and corresponding conversion efficiency as a function of the external PM angle at fixed frequency of 9.14, 9.91, and $10.41 \mathrm{THz}$, respectively. (b) Calculated THz output at 9.41 on the basis of Equations (1)-(4) (broken line), and that result which was taken into account contribution of the interference of each three interacting waves (solid line).

\section{Acknowledgements}

Authors would like to thank the organization of Nishizawa Memorial Research Centre for its support of the GaSe crystal growth. This work was supported by KAKENHI (23360025) of Grant-in-Aid for Scientific Research (B) and KDDI Foundation Grant Programs.

\section{References}

[1] Tonouchi, M. (2007) Cutting-Edge Terahertz Technology. Nature Photonics, 1, 97-105. http://dx.doi.org/10.1038/nphoton.2007.3

[2] Laman, N., Harsha, S.S., Grischkowsky, D. and Melinger, J.S. (2008) High-Resolution Waveguide THz Spectroscopy of Biological Molecules. Biophysical Journal, 94, 1010-1020. http://dx.doi.org/10.1529/biophysj.107.113647

[3] Schaar, J.E., Vodopyanov, K.L. and Fejer, M.M. (2007) Intracavity Terahertz-Wave Generation in a Synchronously Pumped Optical Parametric Oscillator Using Quasi-Phase-Matched GaAs. Optics Letters, 32, 1284-1286. http://dx.doi.org/10.1364/OL.32.001284

[4] Song, Q., Zhao, Y., Redo-Sanchez, A., Zhang, C. and Liu, X. (2009) Fast Continuous Terahertz Wave Imaging System for Security. Optics Communications, 282, 2019-2022. http://dx.doi.org/10.1016/j.optcom.2009.02.019

[5] Tomita, I., Suzuki, H., Ito, H., Takenouchi, H., Ajito, K., Rungsawang, R. and Ueno, Y. (2006) Terahertz-Wave Generation from Quasi-Phase-Matched GaP for $1.55 \mu \mathrm{m}$ Pumping. Applied Physics Letters, 88, 071118. http://dx.doi.org/10.1063/1.2174832

[6] Tanabe, T., Suto, K., Nishizawa, J., Kimura, T. and Saito, K. (2003) Frequency-Tunable High-Power Terahertz Wave Generation from GaP. Journal of Applied Physics, 93, 4610-4615. http://dx.doi.org/10.1063/1.1560573

[7] Dezaki, H., Tanabe, T., Haiyan, J. and Oyama, Y. (2012) Wide Frequency Tunable GaSe Terahertz Emitter under Collinear Phase Matching Condition. Key Engineering Materials, 500, 58-61. http://dx.doi.org/10.4028/www.scientific.net/KEM.500.58

[8] Nishizawa, J.-I., Sasaki, T., Oyama, Y. and Tanabe, T. (2007) Aspects of Point Defects in Coherent Terahertz-Wave Spectroscopy. Physica B, 401-402, 677-681. http://dx.doi.org/10.1016/j.physb.2007.09.050

[9] Shi, W. and Ding, Y.J. (2004) A monochromatic and High-Power Terahertz Source Tunable in the Ranges of 2.7 - 38.4 and 58.2 - $3540 \mu \mathrm{m}$ for Variety of Potential Applications. Applied Physics Letters, 84, 1635-1637.

[10] Dmitriev, V.G., Gurzadyan, G.G. and Nikogosyan, D.N. (1997) Handbook of Nonlinear Optical Crystals. Springer, Berlin, 166-169.

[11] Cardetta, V.L., Mancini, A.M. and Rizzo, A. (1972) Melt Growth of Single Crystal Ingots of GaSe by Bridgman-Stockbarger's Method. Journal of Crystal Growth, 16, 183-185. http://dx.doi.org/10.1016/0022-0248(72)90112-1

[12] Singh, N.B., Suhre, D.R., Balakrishna, V., Marable, M. and Meyer, R. (1998) Far-Infrared Conversion Materials: Gallium Selenide for Far-Infrared Conversion Applications. Progress in Crystal Growth and Characterization of Materials, 37, 47-102. http://dx.doi.org/10.1016/S0960-8974(98)00013-8

[13] Onai, T., Nagai, Y., Dezaki, H. and Oyama, Y. (2013) Liquid Phase Growth of Bulk GaSe Crystal Implemented with 
the Temperature Difference Method under Controlled Vapor. Journal of Crystal Growth, 380, 18-22. http://dx.doi.org/10.1016/j.jcrysgro.2013.05.027

[14] Nagai, Y., Maeda, K., Suzuki, K. and Oyama, Y. (2014) Comparative Study of Shallow Acceptor Levels in Non-Intentionally Doped P-Type GaSe Crystals Prepared by the Bridgman and Liquid Phase Solution Growth Methods. Journal of Electronic Materials, 43, 3117-3120.

[15] Chen, C.W., Tang, T.T., Lin, S.H., Huang, J.Y., Chang, C.S., Chung, P.K., Yen, S.T. and Pan, C.L. (2009) Optical Properties and Potential applications of $\varepsilon-G a S e$ at Terahertz Frequencies. Journal of Optical Society of America B, 26, A58-A65. http://dx.doi.org/10.1364/JOSAB.26.000A58

[16] Kauppinen, J., Kärkkäinen, T. and Kyrö, E. (1978) High-Resolution Spectrum of Water Vapor between 30 and 720 $\mathrm{cm}^{-1}$. Journal of Molecular Spectroscopy, 71, 15-45. http://dx.doi.org/10.1016/0022-2852(78)90073-5

[17] Ragam, S. (2010) CW THz Wave Generation with Diode Laser Pumping. Ph.D. Thesis, Tohoku University, Sendai. 
Scientific Research Publishing (SCIRP) is one of the largest Open Access journal publishers. It is currently publishing more than 200 open access, online, peer-reviewed journals covering a wide range of academic disciplines. SCIRP serves the worldwide academic communities and contributes to the progress and application of science with its publication.

Other selected journals from SCIRP are listed as below. Submit your manuscript to us via either submit@scirp.org or Online Submission Portal.
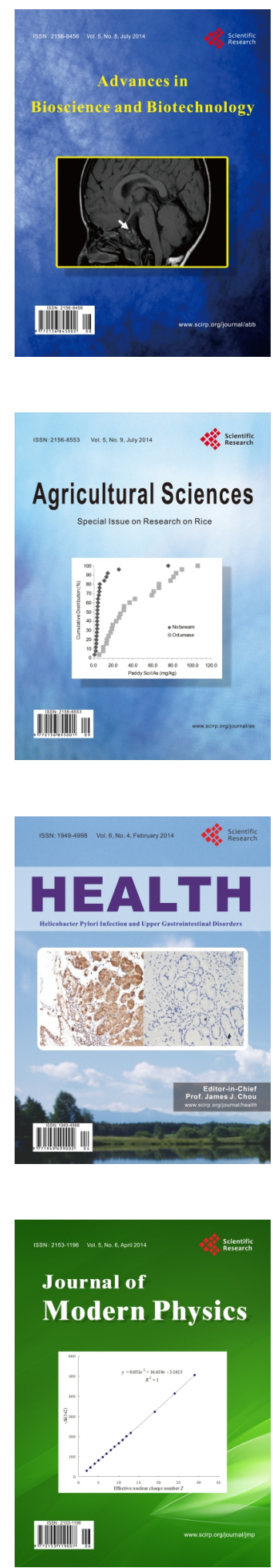
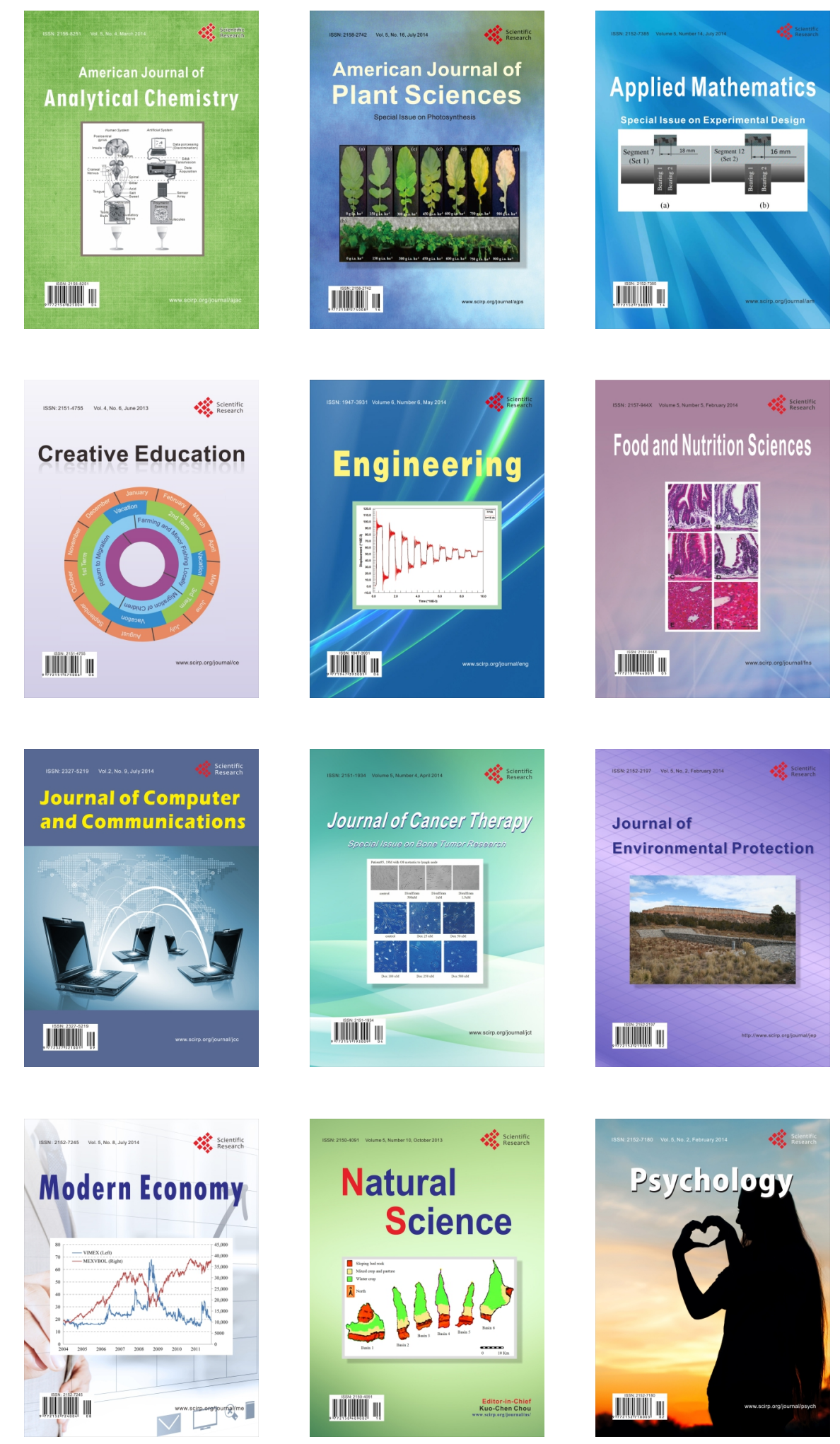